Acta Cryst. (1972). A 28, 170

\title{
Irrelevance of Atomic Masses for Debye-Waller $B$ Values in the Limit of High Temperatures
}

\author{
By C. Huiszoon AND P.P. M. GroeneWEGEN* \\ Chemical Physics Laboratory, Twente University of Technology, Enschede, The Netherlands
}

(Received 24 May 1971)

\begin{abstract}
An expression that approximates Debye-Waller $B$ values by a sum of three terms is derived from the theory of lattice dynamics in the harmonic approximation. For cubic crystals $\left(M_{\kappa}\right.$ is the mass of the $\kappa$ th atom in the unit cell):

$$
B_{\kappa}=\alpha_{\kappa} T+2 \pi^{2} \hbar^{2} / 3 k T M_{\kappa}+\gamma_{\kappa} / M^{2} T^{3},
$$

where $T \gtrsim \Theta_{D} / 2$ and $\alpha_{\kappa}$ and $\gamma_{\kappa}$ are constants, depending on interatomic forces only. It is shown that for temperatures above the Debye temperature $\Theta_{D}$ of the lattice, the second and third terms in the above expression can be neglected. From this, it follows that above the Debye temperature Debye-Waller $B$ values become independent of the atomic masses. Consequently, the heavier atoms in a lattice do not necessarily have the smaller $B$ values.
\end{abstract}

\section{Introduction}

In a crystal lattice, heavier atoms do not necessarily have the smaller Debye-Waller $B$ values. Examples are $\mathrm{PbTe}$ (Keffer, Hayes \& Bienenstock, 1968), MgO (Raccah \& Arnott, 1967; Sanger, 1969), and AgCl (Korhonen \& Linkoaho, 1966). In these cases, roomtemperature Debye-Waller $B$ values of the heavier atoms $(\mathrm{Pb}, \mathrm{Mg}$, and $\mathrm{Ag})$ are larger than those of the lighter atoms $(\mathrm{Te}, \mathrm{O}$, and $\mathrm{Cl}$ ). The phenomenon has been appreciated as rather surprising.

With the aid of the theory of lattice dynamics in the harmonic approximation, a theorem will be derived which states that Debye-Waller $B$ values of individual atoms in a crystal lattice do not depend on the atomic masses when the temperature of the crystal is above its Debye temperature.

For temperatures above half the Debye temperature, an expression is given that allows calculation of $B$ values at any temperature above $\Theta_{D} / 2$ if Debye-Waller values at least one temperature in the region are known (e.g. from experiment).

It is hoped that the theorem will contribute to a qualitative understanding of Debye-Waller $B$ values.

\section{Derivation of theorem}

The vibrations of an atom in a crystal lattice, moving in a harmonic potential field, can be characterized by a symmetric tensor $\mathbf{U}$, with six independent components. The mean-square amplitude of vibration in the direction of a unit vector $\mathbf{l}$, with components $l_{\alpha}$, is given by (Cruickshank, 1956):

$$
\left\langle u^{2}\right\rangle=\sum_{\alpha, \beta}\left\langle u_{\alpha} u_{\beta}\right\rangle l_{\alpha} l_{\beta}
$$

\footnotetext{
* Present address: Philips Natuurkundig Laboratorium, WAALRE, The Netherlands.
}

To evaluate the quantities $\left\langle u_{\alpha} u_{\beta}\right\rangle$, we adopt formulae and notation of lattice dynamics as presented by Maradudin, Montroll \& Weiss (1963).

Eigenvectors and eigenfrequencies, of the phonons present in a lattice, obey the following set of equations:

$$
\sum_{\kappa^{\prime}, \beta} D_{\alpha \beta}\left(\begin{array}{l}
\mathbf{k} \\
\kappa \kappa^{\prime}
\end{array}\right) \mathbf{e}_{\beta}\left(\left.\kappa^{\prime}\right|_{j} ^{\mathbf{k}}\right)=\mathbf{e}_{\alpha}\left(\left.\kappa\right|_{j} ^{\mathbf{k}}\right) \omega_{j}^{2}(\mathbf{k}),
$$

where $\alpha$ and $\beta$ are again the Cartesian coordinates $(x, y, z), \kappa$ and $\kappa^{\prime}$ are indices to number the atoms in the unit cell, $\mathbf{k}$ is the wave vector, $\omega_{j}(\mathbf{k})$ is the eigenfrequency of the $j$ th branch, and $\mathbf{e}\left(\left.\kappa\right|_{j} ^{\mathbf{k}}\right)$ is the eigenvector of the $\kappa$ th atom, belonging to the $j$ th branch. The $r$ atoms in the unit cell give $3 r$ phonon branches. The quantities $D_{\alpha \beta}\left(\kappa_{\kappa}^{\mathbf{k}}\right)$ are the elements of the dynamical matrix:

$$
D_{\alpha \beta}\left(\underset{\kappa \kappa^{\prime}}{\mathbf{k}}\right)=\left(M_{\kappa} M_{\kappa^{\prime}}\right)^{-1 / 2} \sum_{l^{\prime}} \varphi_{\alpha \beta}\left(\begin{array}{ll}
l k^{\prime} \\
k^{\prime}
\end{array}\right) \exp \left[-2 \pi i \mathbf{k} \cdot \mathbf{x}\left(l l^{\prime}\right)\right],
$$

where $M_{\kappa}$ is the mass of the $\kappa$ th atom, $l$ and $l^{\prime}$ are indices to number unit cells, $\mathbf{x}\left(l l^{\prime}\right)=\mathbf{x}(l)-\mathbf{x}\left(l^{\prime}\right)$, and $\mathbf{x}(l)$ is the position of the $l$ th unit cell, while $\varphi_{\alpha \beta}\left(\begin{array}{l}l k_{k \kappa^{\prime}}^{\prime \prime} \\ )\end{array}\right)$ is the negative of the force exerted in the $\alpha$ direction on atom $\left(\begin{array}{l}l \\ k\end{array}\right)$, when atom $\left(\begin{array}{l}l^{\prime} \\ \kappa^{\prime}\end{array}\right)$ is displaced a unit distance in the $\beta$ direction. The eigenvalue equation (2) can be written more compactly in matrix form:

$$
\mathbf{D}(\mathbf{k}) \mathbf{E}(\mathbf{k})=\mathbf{E}(\mathbf{k}) \Lambda(\mathbf{k}) .
$$

From this, it follows that

$$
\mathbf{D}^{-1}(\mathbf{k})=\mathbf{E}(\mathbf{k}) \Lambda^{-1}(\mathbf{k}) \mathbf{E} \dagger(\mathbf{k}) .
$$

The matrix $\mathbf{D}(\mathbf{k})$ is Hermitian; hence, $\mathbf{E}(\mathbf{k})$ is unitary and we obtain from equation (5):

$$
\left(\mathbf{D}^{-1}\right)_{\alpha \beta}\left(\underset{\kappa \kappa^{\prime}}{\mathbf{k}}\right)=\sum_{j} \mathbf{e}_{\alpha}\left(\left.\kappa\right|_{j} ^{\mathbf{k}}\right) \mathbf{e}_{\beta}^{*}\left(\left.\kappa^{\prime}\right|_{j} ^{\mathbf{k}}\right) \omega_{j}^{-2}(\mathbf{k}) .
$$


At this point, we consider the mean products of vibrational amplitudes of the $\left(\begin{array}{l}l \\ \kappa\end{array}\right)$ th atom (Maradudin et al., 1963, p. 237):

$$
\begin{aligned}
\left\langle u_{\alpha}\left(\begin{array}{l}
l \\
k
\end{array}\right) u_{\beta}\left(\begin{array}{l}
l \\
k
\end{array}\right)\right\rangle=\frac{\hbar}{2 N M_{\kappa}} \sum_{\kappa, j} & \mathbf{e}_{\alpha}(\kappa \mid \mathbf{j}) \mathbf{e}_{\beta}^{*}\left(\left.\kappa\right|_{j} ^{\mathbf{k}}\right) \\
& \times \frac{\operatorname{coth}\left[\hbar \omega_{j}(\mathbf{k}) / 2 k T\right]}{\omega_{j}(\mathbf{k})},
\end{aligned}
$$

where $N$ is the number of unit cells in the crystal. The summation is carried out over $N$ values of the wave vector $\mathbf{k}$, distributed through the first Brillouin zone, and, for each value of the wave vector $\mathbf{k}$, over the $3 r$ phonon branches. Following Waller (1925), we expand the hyperbolic cotangent:

$$
\operatorname{coth} \frac{\hbar \omega}{2 k T}=\frac{2 k T}{\hbar \omega}+\frac{\hbar \omega}{6 k T}-\frac{\hbar^{3} \omega^{3}}{360 k^{3} T^{3}} \ldots, \frac{\hbar \omega}{2 k T}<1
$$

and obtain from equation (7):

$$
\begin{aligned}
\left\langle u_{\alpha}\left(\begin{array}{l}
l \\
k
\end{array}\right) u_{\beta}\left(\begin{array}{l}
l \\
k
\end{array}\right)\right\rangle & =\frac{\hbar}{2 N M_{\kappa}} \sum_{\mathbf{k}, j} \mathbf{e}_{\alpha}\left(\left.\kappa\right|_{j} ^{\mathbf{k}}\right) \mathbf{e}_{\beta}^{*}\left(\left.\kappa\right|_{j} ^{\mathbf{k}}\right) \\
& \times\left[2 k T / \hbar \omega_{j}(\mathbf{k})+\hbar \omega_{j}(\mathbf{k}) / 6 k T\right. \\
& \left.-\hbar^{3} \omega_{j}^{3}(\mathbf{k}) / 360 k^{3} T^{3}\right] \omega_{j}^{-1}(\mathbf{k}) .
\end{aligned}
$$

The condition $\hbar \omega_{j}(\mathbf{k}) / 2 k T<1$ is roughly equivalent to $T>\Theta_{D} / 2, \Theta_{D}$ being the Debye temperature of the lattice. $\Theta_{D}$ is connected with the maximum phonon frequency, $\omega_{m}$, as $\hbar \omega_{m} / k \simeq \Theta_{D}$. The second and third terms of the expression between square brackets of equation (9) contribute well below $8 \%$ when $T \gtrsim \Theta_{D}$. Neglecting these terms, equation (9) can be written as:

$$
\left\langle u_{\alpha}\left(\begin{array}{l}
l \\
k
\end{array}\right) u_{\beta}\left(\begin{array}{l}
l \\
k
\end{array}\right)\right\rangle=\frac{k T}{N M_{\kappa}} \sum_{\mathbf{k}, j} \mathbf{e}_{\alpha}\left(\left.\kappa\right|_{j} ^{\mathbf{k}}\right) \mathbf{e}_{\beta}^{*}\left(\left.\kappa\right|_{j} ^{\mathbf{k}}\right) \omega_{j}^{-2}(\mathbf{k})
$$

and with equation (6):

$$
\left\langle u_{\alpha}\left(\begin{array}{l}
l \\
\kappa
\end{array}\right) u_{\beta}\left(\begin{array}{l}
l \\
\kappa
\end{array}\right)\right\rangle=\frac{k T}{N M_{\kappa}} \sum_{\mathbf{k}}\left(\mathbf{D}^{-1}\right)_{\alpha \beta}\left(\left(\begin{array}{c}
\mathbf{k} \\
\kappa \kappa
\end{array}\right) .\right.
$$

In order to proceed we recognize that:

$$
\begin{aligned}
\mathbf{D}(\mathbf{k}) & =\mathbf{M}^{-1 / 2} \Phi(\mathbf{k}) \mathbf{M}^{-1 / 2}, \\
D_{\alpha \beta}(\underset{\kappa \kappa}{\mathbf{k}}) & =M_{\kappa}^{-1} \boldsymbol{\Phi}_{\alpha \beta}(\underset{\kappa \kappa}{\mathbf{k}}), \\
\mathbf{D}^{-1}(\mathbf{k}) & =\mathbf{M}^{1 / 2} \boldsymbol{\Phi}^{-1}(\mathbf{k}) \mathbf{M}^{1 / 2}, \\
\left(\mathbf{D}^{-1}\right)_{\alpha \beta}\left({ }_{\kappa \kappa}^{\mathbf{k}}\right) & =M_{\kappa}\left(\Phi^{-1}\right)_{\alpha \beta}(\underset{\kappa \kappa}{\mathbf{k}}),
\end{aligned}
$$

where $\mathbf{M}$ is a diagonal matrix with $M_{11}=M_{22}=M_{33}=$ $M_{1}$, the mass of atom $1, M_{44}=M_{55}=M_{66}=M_{2}$, the mass of atom 2, and so on. The matrix $\Phi(\mathbf{k})$ is defined by:

$$
\boldsymbol{\Phi}_{\alpha \beta}\left(\begin{array}{c}
\mathbf{k} \\
\kappa \kappa^{\prime}
\end{array}\right)=\sum_{l^{\prime}} \varphi_{\alpha \beta}\left(\begin{array}{l}
l k^{\prime} \kappa^{\prime} \\
l^{\prime}
\end{array}\right) \exp \left[-2 \pi i \mathbf{k} \cdot \mathbf{x}\left(l l^{\prime}\right)\right] .
$$

crystals for temperatures above half the Debye temperature if, at least, one Debye-Waller $B$ value is known in that temperature region, to be calculated. It is emphasized that the elements of $\boldsymbol{\Phi}$ do not depend on the atomic masses. Substitution of equation (13b) in equation (11) gives:

$$
\left\langle u_{\alpha}\left(\begin{array}{l}
l \\
k
\end{array}\right) u_{\beta}\left(\begin{array}{l}
l \\
k
\end{array}\right)\right\rangle=\frac{k T}{N} \sum_{\mathbf{k}}\left(\boldsymbol{\Phi}^{-1}\right)_{\alpha \beta}\left(\begin{array}{l}
\mathbf{k} \\
\kappa \kappa
\end{array}\right), T \gtrsim \Theta_{D} .
$$

Before commenting on equation (15), we turn our attention to the second and third terms of the expression

\begin{tabular}{|c|c|c|c|c|}
\hline Temperature $\left({ }^{\circ} \mathrm{K}\right)$ & $B_{\mathrm{K}} *$ & $B_{\mathrm{Br}} *$ & $B_{\mathrm{K}} \uparrow$ & $B_{\mathrm{Br}} \dagger$ \\
\hline 295 & $2 \cdot 2636$ & $2 \cdot 4584$ & $2 \cdot 2249(98 \cdot 8)$ & $2.4162(99.5)$ \\
\hline 275 & $2 \cdot 1000$ & $2 \cdot 2784$ & $2.0778(98.6)$ & $2 \cdot 2540(99 \cdot 4)$ \\
\hline 255 & 1.9393 & $2 \cdot 1012$ & $1.9308(98.4)$ & $2.0920(99 \cdot 3)$ \\
\hline 235 & $1 \cdot 7811$ & 1.9267 & $1 \cdot 7842(98 \cdot 1)$ & $1.9301(99 \cdot 2)$ \\
\hline 215 & $1 \cdot 6258$ & 1.7549 & $1.6379(97.8)$ & $1.7682(99 \cdot 1)$ \\
\hline 195 & $1 \cdot 4732$ & 1.5859 & $1.4921(97.4)$ & $1.6065(98.9)$ \\
\hline 175 & $1 \cdot 3236$ & 1.4195 & $1 \cdot 3467(96 \cdot 8)$ & $1.4449(98.7)$ \\
\hline 155 & $1 \cdot 1771$ & $1 \cdot 2560$ & $1 \cdot 2021(96 \cdot 1)$ & $1.2833(98.4)$ \\
\hline 135 & 1.0342 & 1.0954 & $1.0579(95 \cdot 1)$ & $1 \cdot 1214(98 \cdot 1)$ \\
\hline 115 & $0 \cdot 8955$ & 0.9382 & $0.9142(93.7)$ & $0.9586(97.7)$ \\
\hline 95 & $0 \cdot 7621$ & 0.7849 & $0.7695(92 \cdot 0)$ & $0.7928(97 \cdot 6)$ \\
\hline 75 & 0.6362 & 0.6367 & $0.6183(90.4)$ & $0.6172(99.0)$ \\
\hline
\end{tabular}
between square brackets of equation (9). These terms become important when the temperature of the crystal is below its Debye temperature. To evaluate the contributions due to these terms, we use the following properties of the eigenvectors (Maradudin et al., 1963, p. 12):

$$
\begin{gathered}
\sum_{j} \mathbf{e}_{\alpha}\left(\left.\kappa\right|_{j} ^{\mathbf{k}}\right) \mathbf{e}_{\beta}^{*}\left(\left.\kappa\right|_{j} ^{\mathbf{k}}\right)=\delta_{\alpha \cdot \beta}, \\
\sum_{j} \omega_{j}^{2}(\mathbf{k}) \mathbf{e}_{\alpha}\left(\left.\kappa\right|_{j} ^{k}\right) \mathbf{e}_{\beta}^{*}\left(\left.\kappa\right|_{j} ^{\mathbf{k}}\right)=D_{\alpha \beta}\left(\begin{array}{c}
\mathbf{k} k \\
\kappa \kappa
\end{array}\right) .
\end{gathered}
$$

Recalling that the number of $\mathbf{k}$ vectors equals $N$, we find from equations (9), (12a), (15), (16) and (17):

Table 1. Calculated Debye-Waller B values of $\operatorname{KBr}\left(\AA^{2}\right)$.

The contribution from the first term, which is independent of atomic masses, is indicated by $\%$ in parentheses.

* Calculated by Reid \& Smith (1970).

$\uparrow$ Recalculated with equation (19). The two sums have been evaluated indirectly by least-squares fit of equation (19) to the Reid \& Smith data for the 12 temperatures indicated in the first column. 


$$
\begin{array}{r}
\left\langle u_{\alpha}\left(\begin{array}{l}
l \\
k
\end{array}\right) u_{\beta}\left(\begin{array}{l}
l \\
k
\end{array}\right)\right\rangle=\frac{k T}{N} \sum_{\mathbf{k}}\left(\Phi^{-1}\right)_{\alpha \beta}\left(\begin{array}{l}
\mathbf{k} \kappa \\
\kappa \kappa
\end{array}\right)+\frac{\hbar^{2} \delta_{\alpha \beta}}{12 k T M_{\kappa}} \\
-\frac{\hbar^{4}}{720 k^{3}} \overline{T_{N}^{3} M_{\kappa}^{2}} \sum_{\mathbf{k}} \Phi_{\alpha \beta}\left(\begin{array}{l}
\mathbf{k} \\
\kappa \kappa
\end{array}\right), T>\Theta_{D} / 2 .
\end{array}
$$

Equation (18) describes the vibration of an atom in a crystal lattice for temperatures above half the Debye temperature of the lattice. From the quantities $\left\langle u_{\alpha}\left(\begin{array}{l}l \\ k\end{array}\right) u_{\beta}\left(\begin{array}{l}l \\ k\end{array}\right)\right\rangle$, the anisotropic Debye-Waller $B$ values can be obtained by well known methods (Cruickshank, 1956). At high temperatures, $T>\Theta_{D}$, only the first term in the right-hand part of equation (18) is of importance, and Debye-Waller $B$ values become independent of the atomic masses.

Two restrictions should be made. Equation (18) has been derived within the harmonic approximation which will certainly be violated at very high temperatures. The second restriction is in dealing with the temperature dependence of the two sums in equation (18). The matrix elements $\left(\Phi^{-1}\right)_{\alpha \beta}\left(\begin{array}{c}\mathbf{k} \\ k \kappa\end{array}\right)$ and $\Phi_{\alpha \beta}\left(\begin{array}{l}\mathbf{k} k \\ k \kappa\end{array}\right)$ are temperature dependent, via the interatomic forces which depend, for example, on the atomic distances. It is expected, however, that the sums will vary only very little with temperature.

\section{Example}

For a cubic lattice, the Debye-Waller $B$ value of the $\kappa$ th atom is obtained from equation (18) as:

$$
\begin{aligned}
B_{\kappa}=8 \pi^{2}\left[\frac{k T}{N} \sum_{\mathbf{k}}\left(\boldsymbol{\Phi}^{-1}\right)_{\alpha \alpha}\left(\begin{array}{l}
\mathbf{k} \kappa \\
\kappa \kappa
\end{array}\right)+\frac{\hbar^{2}}{12 k T M_{\kappa}}\right. \\
\left.-\frac{\hbar^{4}}{720 k^{3} T_{N}^{3}} M_{\kappa}^{2} \cdot \sum_{\mathbf{k}} \Phi_{\alpha \alpha}\left(\begin{array}{l}
\mathbf{k} \kappa \\
\kappa \kappa
\end{array}\right)\right] .
\end{aligned}
$$

In the above expression the first term in the right-hand part is independent of the atomic mass. The other two terms are inversely proportional to the atomic mass and to the square of the atomic mass. In general, it is very difficult to evaluate the two sums of equation (19), because, for this, a detailed knowledge of the atomic forces is required. We have determined the two sums by a least-squares fit of equation (19), for 12 temperatures, to $\mathrm{KBr} B$ values calculated by Reid \& Smith (1970). The Debye temperature of $\mathrm{KBr}$ is about $160^{\circ} \mathrm{K}$ (Reid \& Smith, 1970) and the 12 temperatures chosen range from $75^{\circ} \mathrm{K}$ (about half the Debye temperature) up to $295^{\circ} \mathrm{K}$. Results are shown in Table 1 .

The results in Table 1 show the various contributions to $B_{\kappa}$ at temperatures above the Debye temperature (for $\mathrm{KBr} 160^{\circ} \mathrm{K}$ ) the main contribution to the DebyeWaller $B$ values comes from the mass-independent term of equation (19). Table 1 also shows that equation (19) describes very well, in a large temperature region, the temperature dependence of both the DebyeWaller $B$ values of $\mathrm{KBr}$.

\section{References}

Cruickshank, D. W. J. (1956). Acta Cryst. 9, 747.

Keffer, C., Hayes, T. M. \& Bienenstock, A. (1968). Phys. Rev. Letters, 21, 1676.

Korhonen, U. \& Linkoaho, M. (1966). Ann. Acad. Sci. Fenn. A VI, No. 195.

Maradudin, A. A., Montroll, E. W. \& Weiss, G. H. (1963). Theory of Lattice Dynamics in the Harmonic Approximation. New York: Academic Press.

Raccah, P. M. \& Arnott, R. J. (1967). Phys. Rev. 153, 1028.

ReID, J. S. \& Smith, T. (1970). J. Phys. Chem. Solids, 31, 2689.

Sanger, P. L. (1969). Acta Cryst. A25, 694.

WALLER, I. (1925). Dissertation, p. 27. Uppsala.

Acta Cryst. (1972). A28, 172

\title{
The Method of Ascent in Symmetry. I. Theory and Tables
}

\author{
By L.L. BoyLE \\ University Chemical Laboratory, Canterbury, Kent, England
}

(Received 3 December 1970)

\begin{abstract}
Supergroup tables are presented whereby a representation of a subgroup can be correlated with those representations of the supergroup which are obtained on ascent in symmetry. The method of derivation is explained and various orientations of the subgroup with respect to the supergroup considered. The tables also include the correlations between the double-valued representations of the corresponding double groups.
\end{abstract}

\section{Introduction}

The well-known process of descent in symmetry allows one to discuss how the representations of a given group decompose into representations of a subgroup. Tables have been constructed to facilitate many such correlations and these are very useful in numerous physical problems, e.g. the splitting of atomic energy levels in a crystal field.

The reverse correlation, in which we ascend in 